\title{
Denoising Analysis on UWB Communication in Mine Tunnels
}

\author{
ZHANG Hongwei \\ Management Training Center of HeiLongJiang Electric Power Limited Company \\ Harbin 150030, China \\ Zh_hongclj@163.com
}

\begin{abstract}
In the EMC interference environment of mine, we found the EMC is white noise and pulse interference. The UWB signal pulses is short and signal power spectral density distributed in wide band is very low. This article mainly proposes a de-noising method of Wavelet transform modulus maxima in signal band, focused on the traditional low-pass filter that can only remove the outside-band noise. Simulation results show that the scheme can effectively retain the signal singularity based on information to achieve obvious noise reduction
\end{abstract}

Keywords- wavelet analysis, denoise, UWB, mine

\section{INTRODUCTION}

According to the coal mine enterprise's production characteristics, two sets of main fan in air shaft is the oversize load key equipment of mine production. Responsibility to the underground mine conveying fresh air, exhausting harmful gas, guarantee the safety of mine production task. At the same time, because the main fan working in the dirty air, voltage is higher, and the current failure probability are large, they are recessive, unpredictable, serious harm to production. The existences of hidden faults seriously affect all the economic and safe operation. Therefore, design a set of perfect function, safety and reliability, convenient operation of the mine main fan on line monitoring system of main fan operation, control, and the various parameters and operating characteristics of timely, accurate monitoring, to ensure mine safety and raise economic efficiency is important.

\section{Mine TUNNEL NOISE ANALYSIS}

According to the analysis of mine tunnels electromagnetic radiation disturbance data [1], transmission line in tunnels is symmetrical three-phase, and it basically does not produce electromagnetic radiation interference. Therefore, the major interference in tunnels is the electromagnetic radiation disturbance from Monitoring Substation gap leakage. On the other hand, the extensive using of contact net of electric locomotive, the contact net electromagnetic disturbance in addition to having the power cable electromagnetic disturbance of all properties, also has the characteristics of random pulse. In the mining area of the steel rail loading point, basic have been filled by coal, easy to produce electric spark interference. Another kind of interference is the main discharge spark and relatively large $d t / d i$ formed within the electromagnetic radiation disturbance in tunnel.
Due to the underground noise analysis above, we can get a conclusion. The noise is mainly divided into the background of Gauss white noise as figure 1 and impulse noise as figure 2. Mine noise interference in the mathematical model can be expressed as follow,

$$
W_{\text {noise }}=W_{a}+W_{t}
$$

In formula, $W_{a}$ is the background of Gauss white noise, mean value is 0 , variance is $\sigma_{a} . W_{t}$ is impulse noise.

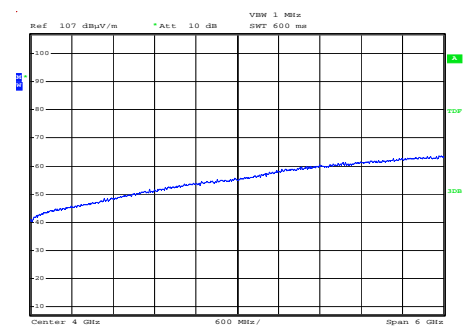

Fig.1 The electromagnetic interference of the electrical and chamber port and a monitor sub-station in the coal mine

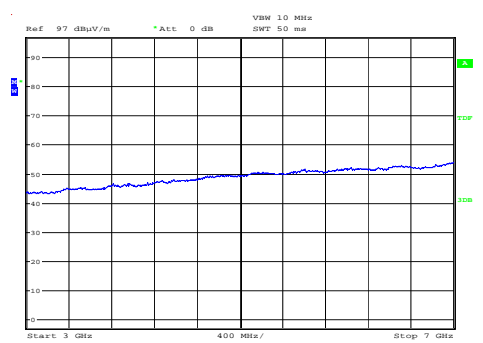

Fig.2 The electromagnetic interference when the motor vehicle wiring running of roadway

\section{DENOISE PRINCIPLE}

Usually the main filtering method we use is the smooth communication signal Fourier transform, the signal in the time domain features are mapped to the frequency domain analysis, and through the band filter to achieve noise reduction [2]. However, when the received signal with noise is large enough, receiving system cannot work. The UWB pulse signal has a great duty ratio, has obvious singularity, very suitable for the singularity analysis to remove the inband noise.

In theory, the singularity of signal can be analyzed by wavelet modulus maxima [3], wavelet transform modulus maxima at different scales to measure the attenuation speed will be used to describe the singular properties of singular 
index. Due to white noise random distribution and the singularity exponent is negative, so the modulus maxima in the binary scale does not possess transitivity.

$$
\begin{aligned}
& E\left[\left|W_{n}(s, x)\right|^{2}\right]=\int_{-\infty}^{+\infty} \int_{-\infty}^{+\infty} E\left[n(u) n(r) \psi_{s}(s-u) \psi_{s}(x-r) d u d r\right] \\
& d_{s}=\frac{1}{s \pi}\left(\frac{\left\|\psi^{(2)}\right\|_{2}}{\left\|\psi^{(1)}\right\|_{2}}+\frac{\left\|\psi^{(1)}\right\|_{2}}{\|\psi\|_{2}}\right)
\end{aligned}
$$

White noise $\mathrm{n}(\mathrm{S})$ with zero mean, $\mathrm{w}(\mathrm{s}, \mathrm{x})$ is the wavelet transform coefficients of white noise in S scales. They are the wavelet first order and Nikai Mi function. According to the formula above, the absolute of noise control module maximum value decreases rapidly with the scale of the increase of S. With the increasing of S, at least half of the modulus maxima can transfer to a larger size. Therefore, from the small scale can be thought of not delivered to large scale modulus maximum is control by noise [4]. So, we can remove these maximums, this will retain the modulus maxima reconstruction of the original signal, suppress the in-band noise effect.

\section{SIMULATION AND ANALYSIS}

\section{A. Analysis of UWB Performanc}

In the UWB wireless communication system, due to the provisions of FCC, on the ground of indoor systems, it is required to select Gauss pulse five derivative waveform at least to meet the above FCC spectrum and power limit requirements. But in underground by using the technology of UWB, does not exist for other narrow-band interference, and can therefore not limited by the ground condition, can reduce the system design requirements, and use the low order Gauss pulse wave communication.

Considering the interference is more serious, the system uses a binary code which has bit error performance advantages of the BPSK modulation mode, and a single cycle of first-order Gauss pulse as ultra broadband emission waveforms. Due to the differential effect of transmit and receive antennas, send signal is one step pulse, the received signal is two order derivative.

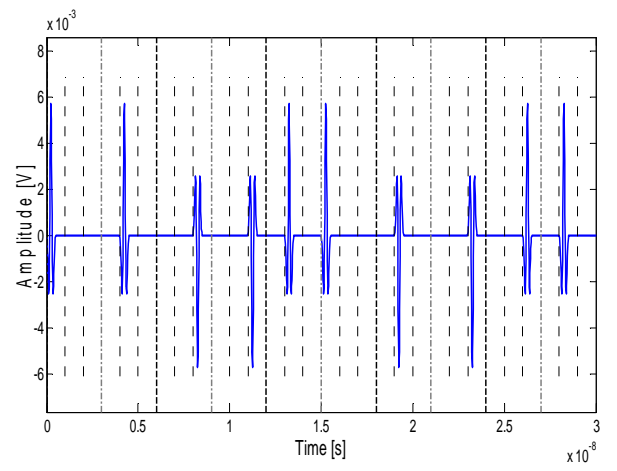

Fig3 TH-UWB received signal

\section{B. Band filter}

Based on analysis noise in mine tunnels, underground noise mainly is the background of Gauss white noise and impulse noise. Therefore, the signal-to-noise ratio is $-30 \mathrm{~dB}$ noisy signals as an example, we use the band pass filter to filter through the receiving antenna. With the Blackman window function design is used, it is 168 order filter and band-pass within the range of $0 \sim 10 \mathrm{GHz}$, transition bandwidth of $1.5 \mathrm{GHz}$, the stop band and a maximum of 0.05 pass band ripple. Through the simulation analysis, band-pass filter can filter out the noise outside of the band only, and the band noise is still existence.

\section{Basic steps In band denoise}

The received signal with noise is removed by wavelet analysis to the outside of the belt filter preprocessing signal [5].

1) Wavelet decomposition process

Linear phase and orthogonal properties of DB4 wavelet is selected when external noise signal wavelet analysis have been done.

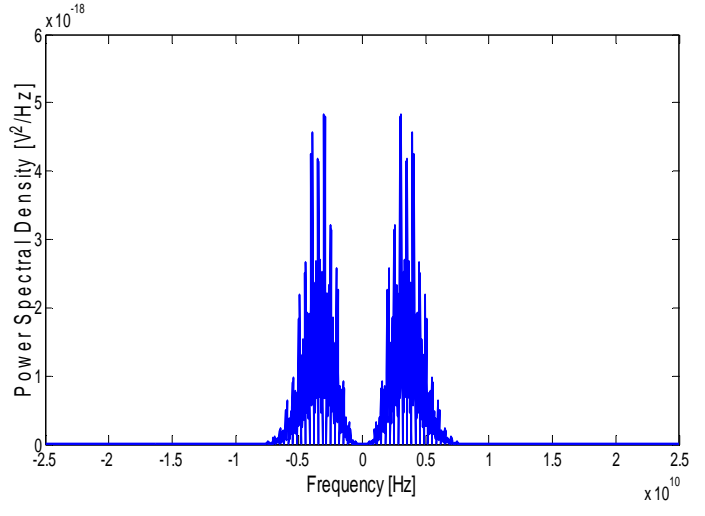

Fig4 clean signal de-noising by lowpass filter

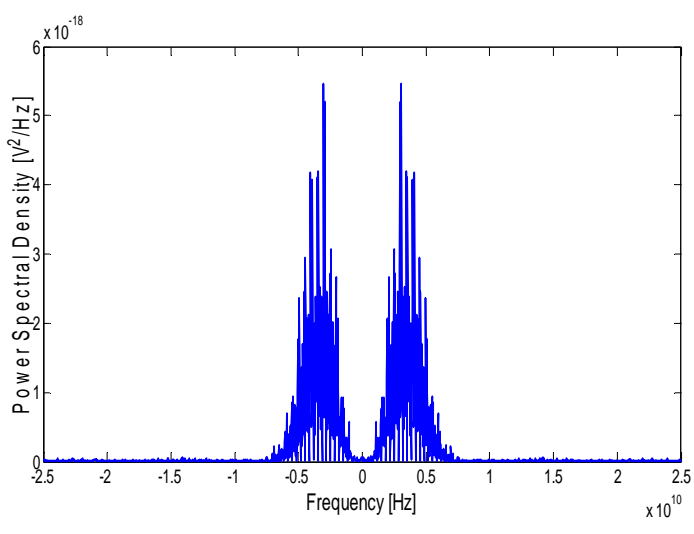

Fig5 signal with noise de-noising by low-pass filter 


\section{2) Scale threshold selection}

Beginning from the maximum value of $s$, we choose threshold amplitude and retain absolute value larger than the threshold value of the modulus maxima as information point, then information corresponding will be found to the transmitted bit in less than the threshold value scale.

3) Analysis of the results

Considering the mine tunnels are the low SNR environment, this research first filters signal with noise band filter. As the simulation results shown that the effects of noisy signal in band filtering are mainly up to signal singularity. Large scale wavelet coefficient will lose the information of signal correlation singular point, and the small scales of wavelet coefficients will more easily affected by noise and produce the extreme point. The results are shown in Fig.6 and Fig.7.

\section{CONCLUSION}

The traditional filtering scheme is only reduce signal noise band, and the mine tunnels environment noise pollution is more serious. In order to ensure a good communication quality, the signal band filtering problem must be solved. This paper discusses the traditional low

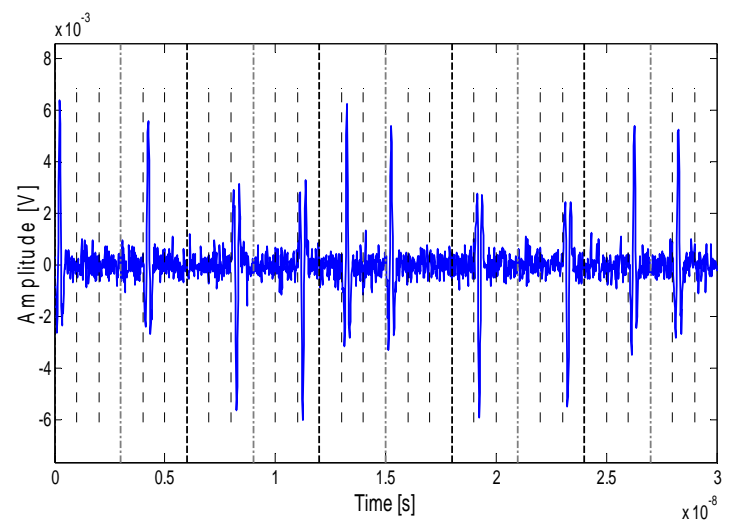

Fig6 signal with low-pass pass filtering method based on the modulus maxima of wavelet filtering method, improves the low SNR performance of communication, but this method of signal reconstruction is more complex, and there are a certain amount of waveform distortion, and reasonable threshold values are to be studied.

\section{REFERENCES}

[1] SUN Jiping, WANG Fuzeng, "Analysis of Affection of Electromagnetic Interference on Communication and Monitoring and Control System in Coal Mine Underground," [J].Industry and Mine Automation, 2009(2).

[2] LI Yu-feng, "Bayesian Denoising for Remote Sensing Image Based on Undecimated Di screte Wavelet Transform,"[J]. Communication Technology. 2009,42(03): 223-225.

[3] Mallat S, Hwang W L. 'Singularity Detection and Processing with Wavelets," [J]. IEEE Transaction on Information Theory. 1992, 38(02): 667-643.

[4] CHENG Li-zhi, WANG Hong-xia, LUO Yong, "Wavelet Theory and Application,”[M]. BEIJING, Science Press,2004.

[5] YUE Li ,WANG Yu-dong,ZHU Xiao-ming. "Computer simulation based on wavelet denoising in DS-UWB," [J]. Journal of Changchun University, 2007, 17(05), 63-104.

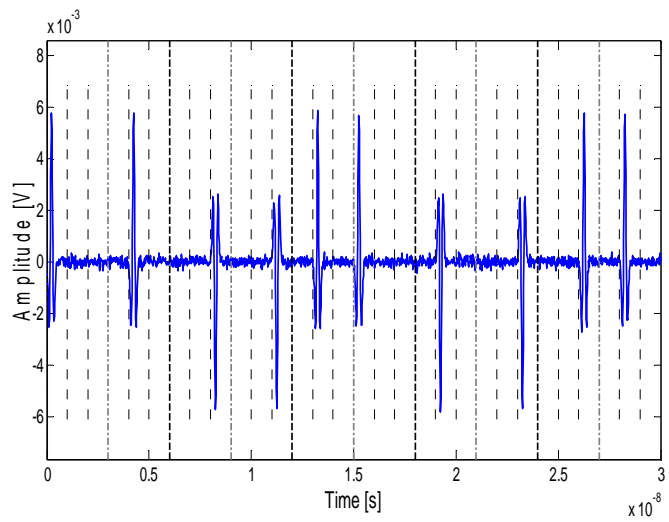

Fig7 signal with low-pass and wavelet filtering 